\title{
Safer glucocorticoid therapy-Bax to the future?
}

Glucocorticosteroids used to treat both adult and pediatric rheumatic diseases are known to have adverse effects on bone, which can impair growth in children and contribute to development of osteoporosis. New data from Farasat Zaman and colleagues, at the Karolinska Institute, Sweden, suggest that the proapoptotic protein Bax has a key role in the chondrocyte dysfunction underlying these issues.

"We found that mice lacking Bax are totally resistant to glucocorticoid-induced bone growth impairment," explains Zaman. Furthermore, in dexamethasonetreated wild-type mice, bone growth retardation was associated with decreased proliferation and increased apoptosis of chondrocytes, and reduced matrix content and integrity of growth plates; Bax gene knockout mice were protected from these effects.

Additional in vitro experiments revealed that dexamethasone increased expression and induced a change in the conformation of Bax, resulting in

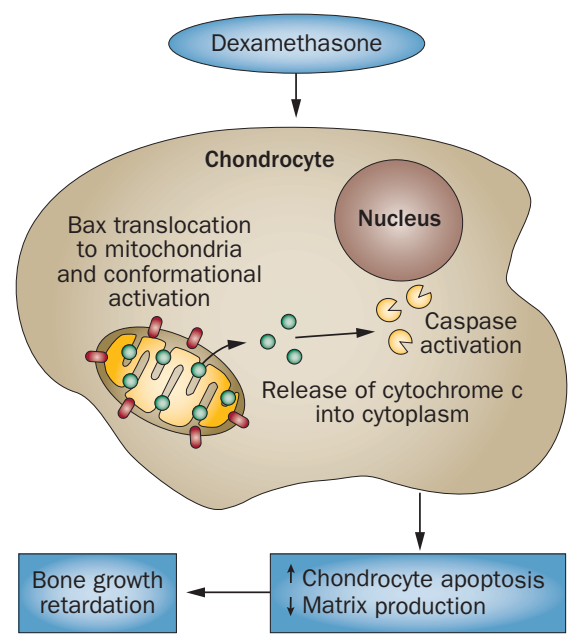

its translocation to mitochondria in chondrocytes. Associated suppression of mitochondrial membrane potential, increased cytochrome $c$ release and resultant chondrocyte apoptosis, were reduced by knockdown of Bax using siRNA. A conformational change in Bax, indicative of its activation, was also identified in growth plate biopsies from tall children treated with dexamethasone to halt their continued bone growth.

"This is the first time that glucocorticoid treatment has been shown to induce conformational changes in Bax in chondrocytes, causing inhibition of cell proliferation and differentiation," claims Zaman. "Based on our data, we speculate that suppression of Bax may provide a new treatment strategy to prevent bone growth disorders," he continues.

Although requiring further research, conceivably, suppression of Bax using small molecules or peptides could also prevent the undesired effects of glucocorticosteroids on bone. "One such peptide is humanin (originally discovered as a neuronal rescue factor in patients with Alzheimer disease), which acts as a negative regulator of Bax," concludes Zaman.

\section{David Killock}

Original article Zaman, F. et al. Ablation of the pro-apoptotic protein Bax protects mice from glucocorticoid-induced bone growth impairment. PLoS ONE 7, e33168 (2012) 